\title{
Perdas de amônia por volatilização em resposta a adubação nitrogenada do feijoeiro
}

\section{Susiane Moura Cardoso dos Santos, João Arthur Antonangelo², Angélica Cristina Fernandes Deus $^{3}$, Dirceu Maximino Fernandes ${ }^{3}$}

\author{
${ }^{1}$ Universidade Estadual de Mato Grosso do Sul - UEMS, Unidade Universitária de Cassilândia, Cassilândia, Mato Grosso do Sul, \\ Brasil. E-mail: susianemoura@yahoo.com.br \\ ${ }^{2}$ Universidade de São Paulo, Escola Superior de Agricultura "Luiz de Queiroz" - ESALQ, Piracicaba, São Paulo. E-mail: \\ joaoantonangelo@usp.br
}

${ }^{3}$ Universidade Estadual Paulista Júlio de Mesquita Filho-UNESP, Faculdade de Ciências Agronômicas de Botucatu, Botucatu, São Paulo. E-mails: angelica_agronomia@yahoo.com.br; dmfernandes@fca.unesp.br.

Recebido: 24/09/2015; Aceito: 14/03/2016.

\section{RESUMO}

O nitrogênio (N) é um dos nutrientes que mais limita o crescimento e o desenvolvimento das plantas, principalmente, devido a sua grande dinâmica no sistema solo-planta-atmosfera. Dentre os vários processos que este elemento está sujeito, pode-se destacar a volatilização. As perdas de amônia $\left(\mathrm{N}-\mathrm{NH}_{3}\right)$ por volatilização podem atingir $78 \%$ do $\mathrm{N}$ aplicado na forma de ureia, e variam de acordo com a fonte utilizada. Esse trabalho teve como objetivo quantificar as perdas de amônia $\left(\mathrm{N}-\mathrm{NH}_{3}\right)$ por volatilização, decorrentes da aplicação de diferentes doses e fontes de $\mathrm{N}$ na cultura do feijão (Phaseolus vulgaris L.). O delineamento experimental utilizado foi o de blocos ao acaso, com dezesseis tratamentos e cinco repetições. Os tratamentos foram constituídos de três doses de $\mathrm{N}$ em cobertura $(100,200$ e $300 \mathrm{mg}$ $\mathrm{dm}^{-3}$ ) e cinco fontes de $\mathrm{N}$, além de um tratamento controle (sem adubação nitrogenada). As maiores perdas de $\mathrm{N}-\mathrm{NH}_{3}$ por volatilização ocorreram na segunda época de aplicação para as fontes Ajifer, ureia e nitrato de amônio.

Palavras-chave: nitrogênio, Phaseolus vulgaris L., adubação, perdas de N.

\section{Ammonia losses by volatilization in response to nitrogen fertilization of bean common crop}

\begin{abstract}
Nitrogen $(\mathrm{N})$ is one of the nutrients that most limits the growth and development of plants, mainly because of its wide dynamics in the soil-plant-atmosphere system. Among the various processes that this element is subject, can highlight volatilization. The ammonia $\left(\mathrm{N}-\mathrm{NH}_{3}\right)$ losses by volatilization can reach $78 \%$ of the $\mathrm{N}$ applied as urea, and it varies according to the source used. This study aimed to quantify the $\mathrm{N}-\mathrm{NH}_{3}$ losses by volatilization, resulting from the application of different doses and sources of $\mathrm{N}$ in bean common crop (Phaseolus vulgaris L.). The experimental design was randomized blocks, with sixteen treatments and five repetitions. The treatments consisted of three rates of $\mathrm{N}$ in cover (100, 200 and $300 \mathrm{mg} \mathrm{dm}^{-3}$ ) and five $\mathrm{N}$ sources, and a control treatment (no nitrogen fertilization). The highest numerical losses of $\mathrm{N}-\mathrm{NH}_{3}$ volatilization occurred in the second period of application for the Ajifer, urea and ammonium nitrate sources.
\end{abstract}

Key words: nitrogen, Phaseolus vulgaris L., fertilization, N losses. 


\section{Introdução}

$\mathrm{O}$ nitrogênio $(\mathrm{N})$ é o nutriente requerido em maiores quantidades pela cultura do feijoeiro. Por ser um elemento que apresenta grande dinâmica no sistema solo-planta-atmosfera pode ser perdido facilmente por volatilização ou lixiviação; além disso, os adubos nitrogenados têm baixa eficiência e alto custo de sintetização, o que permite considerar que sua utilização sem critério, pode onerar os custos do sistema produção e contaminar o meio ambiente. A acentuada disponibilidade de $\mathrm{N}$ às plantas de feijão resulta no alongamento da fase vegetativa o que acarreta perda significativa no potencial produtivo.

O manejo adequado da adubação nitrogenada beneficia o meio ambiente por causar menores níveis de acidificação do solo, eutrofização das águas, poluição do lençol freático e salinização de áreas (BITTENCOURT, 2009). Além disso, beneficia vários segmentos da sociedade: o produtor tem maior produtividade e maior margem de lucro; os agentes técnicos alcançam grandes vendas de insumos e, finalmente, os consumidores são beneficiados pelas melhores características organolépticas dos produtos e, provavelmente, por menor preço de compra. Visando o fortalecimento da cadeia produtiva do feijão, torna-se cada vez mais importante a necessidade de técnicas agronômicas objetivando ganhos em produtividade, assim como a obtenção de um produto com características tecnológicas desejáveis, principalmente com teor adequado de proteína bruta e alta capacidade de hidratação.

A amônia $\left(\mathrm{N}-\mathrm{NH}_{3}\right)$ perdida por volatilização pode ser proveniente da mineralização da matéria orgânica ou do fertilizante aplicado, sendo esse o fenômeno mais intenso mediante aumento do $\mathrm{pH}$ do solo. Essa intensificação do processo de perda de $\mathrm{N}$ pode ocorrer nos estágios reprodutivos, onde há aumento do potencial de volatilização de $\mathrm{N}-\mathrm{NH}_{3}$ devido às mudanças no metabolismo do $\mathrm{N}$ da planta, e também por meio da quebra de proteínas e aminoácidos (BOLOGNA, 2006).

As perdas gasosas são o principal fator de ineficiência do uso dos fertilizantes nitrogenados, pois o $\mathrm{N}$ que poderia ser absorvido e assimilado pelas plantas é perdido na forma de amônia $\left(\mathrm{N}-\mathrm{NH}_{3}\right)$ e de óxidos nitrosos $\left(\mathrm{N}_{2} \mathrm{O}\right)$ para a atmosfera.
Esse tipo de perda pode chegar a $80 \%$ do adubo aplicado, ou seja, em casos extremos a planta consegue utilizar apenas $20 \%$ do $\mathrm{N}$ proveniente do fertilizante. Essas perdas precisam ser bem compreendidas para orientação do adequado manejo de adubação, visando melhorar o aproveitamento dos fertilizantes aplicados (OLIVEIRA, 2008).

A pesquisa com fontes de $\mathrm{N}$ vem ao encontro da necessidade de disponibilização do $\mathrm{N}$ para a nutrição das plantas de feijão, de forma gradual e evitando perdas, já que a formação de nódulos (Rhizobium) para fixação do $\mathrm{N}$ atmosférico, no feijoeiro, não é eficiente para suprir toda a necessidade de $\mathrm{N}$ por essa cultura. Dessa forma, são necessários estudos que possibilitem a redução das perdas, aumentando a eficiência de utilização do $\mathrm{N}$ e, consequentemente, a produtividade do feijoeiro.

Esse trabalho teve como objetivo quantificar as perdas de amônia $\left(\mathrm{N}-\mathrm{NH}_{3}\right)$ por volatilização, decorrentes da aplicação em cobertura de diferentes doses e fontes de $\mathrm{N}$ na cultura do feijão (Phaseolus vulgaris L.).

\section{Material e Métodos}

O experimento foi conduzido em um túnel plástico, no Departamento de Recursos Naturais/Área de Ciência do Solo da Faculdade de Ciências Agronômicas UNESP, Campus de Botucatu - SP (22 $51^{\prime \prime}$ de latitude $\mathrm{S}$ e $48^{\circ} 26^{\prime \prime}$ de longitude W, com altitude de $\left.786 \mathrm{~m}\right)$. A temperatura média anual da região é de $20,5^{\circ} \mathrm{C}$ e umidade relativa do ar de $71 \%$ (MARTINS, 2003).

O solo utilizado foi um Latossolo Vermelho distrófico ( $\mathrm{LVd}$ ) de textura média, coletado na camada superficial de 0-20 $\mathrm{cm}$. A análise química do solo seguiu a metodologia proposta por Raij e Quaggio (2001). Os resultados encontram-se na Tabela 1.

A correção da acidez do solo foi realizada com a aplicação de $104 \mathrm{~g}$ de calcário $(\mathrm{PRNT}=96 \%)$ por vaso com o intuito de elevar a saturação do solo a 70\% (RAIJ et al., 1997). O calcário foi incorporado ao solo com auxílio de uma betoneira. Na adubação de base aplicouse $300 \mathrm{mg}$ de fósforo e $40 \mathrm{mg}$ de potássio; não houve aplicação de nitrogênio.

Tabela 1. Resultados da análise química do solo. Botucatu-SP 2010.

\begin{tabular}{|c|c|c|c|c|c|c|c|c|c|c|c|c|c|c|c|c|}
\hline $\begin{array}{c}\text { pH } \\
\mathrm{CaCl}_{2}\end{array}$ & $\begin{array}{l}\text { М.O. } \\
\mathrm{g} \mathrm{dm}^{-3}\end{array}$ & $\begin{array}{c}P_{\text {resina }} \\
\mathrm{Mg} \mathrm{dm}^{-3}\end{array}$ & $\mathbf{A l}^{+3}$ & $\begin{array}{l}\mathbf{H}+\mathbf{A l} \\
\ldots \ldots \ldots .\end{array}$ & $\begin{array}{c}\mathbf{K} \\
\mathrm{nmol}_{\mathrm{c}}\end{array}$ & $\begin{array}{l}\text { Ca } \\
\mathrm{Im}^{-3} .\end{array}$ & Mg & SB & CTC & $\begin{array}{l}\mathbf{V} \\
\%\end{array}$ & $\mathbf{S}$ & B & $\mathbf{C u}$ & $\begin{array}{l}\mathbf{F e} \\
. . \mathrm{mg}\end{array}$ & $\begin{array}{l}\text { Mn } \\
\mathrm{m}^{-3} \ldots\end{array}$ & $\mathbf{Z n}$ \\
\hline 4 & 20 & 4 & 14 & 77 & 0,8 & 9 & 3 & 12 & 89 & 14 & 15 & 0 & 0,9 & 52 & 0,8 & 0,2 \\
\hline
\end{tabular}

Fonte: Laboratório de Fertilidade do Solo do Departamento de Recursos Naturais da Faculdade de Ciências Agronômicas da UNESP, Campus de Botucatu. 
Os tratamentos foram constituídos por cinco fontes de $\mathrm{N}$ [ureia - $45 \%$ de $\mathrm{N}$, sulfato de amônio - $20 \%$ de $\mathrm{N}$, nitrato de cálcio - $20 \%$ de $\mathrm{N}$, nitrato de amônio - $32 \%$ de $\mathrm{N}$ e Ajifer (fertilizante organomineral: coproduto do glutamato monosódico- $4 \%$ de N)] e por três doses de nitrogênio $\left(100,200\right.$ e $300 \mathrm{mg} \mathrm{dm}^{-3}$, aplicados em cobertura, quando as plantas apresentavam 10, 20 e 30 DAE) e um tratamento controle (sem aplicação de $\mathrm{N}$ em cobertura), sendo um fatorial 3 x $5+1$. Como parcelas experimentais foram utilizados vasos de cimento amianto retangulares com capacidade para $40 \mathrm{~L}$ de solo, totalizando 80 vasos.

No dia 24/02/2010 foi realizada a semeadura do cultivar IAC - Alvorada. As sementes foram tratadas com Thiram $200 \mathrm{~g} \mathrm{~L}^{-1}$, fungicida sistêmico. Foram semeadas dez sementes de feijão em cada vaso, com uma profundidade de $2 \mathrm{~cm}$. A emergência do feijão ocorreu no dia 28/02/2010. No quinto dia após a emergência (DAE) das plântulas, realizou-se o desbaste deixando apenas seis plantas por vaso, as quais serviram para avaliação dos parâmetros estudados. $\mathrm{O} N$ foi aplicado aos 10, 20 e 30 DAE. Após a primeira aplicação dos tratamentos, foram dispostos os coletores em cada vaso, para quantificação da amônia perdida por volatilização.

A avaliação das perdas de $\mathrm{N}-\mathrm{NH}_{3}$ por volatilização foi realizada conforme Nönmik (1973) com algumas adaptações. Utilizou-se absorvedores de espuma de 8,0 $\mathrm{x} 8,0 \mathrm{~cm}$, densidade de $0,02 \mathrm{~g} \mathrm{~cm}^{-3}$, as quais foram embebidas em $11 \mathrm{~mL}$ de ácido fosfórico $(0,05 \mathrm{~N})$. Em seguida, as espumas foram colocadas sobre chapas de PVC de $100 \times 100$ × 2 mm e envolvida por uma camada de polytetrafluoretileno (fita "veda rosca"), permeável à amônia e impermeável à água. Os absorvedores foram deixados em sacos plásticos até o momento de serem colocados nos vasos.
Após a aplicação dos tratamentos, em cada vaso colocou-se um absorvedor apoiado por varetas, com a chapa de PVC voltada para cima, evitando que a amônia presente no ar fosse captada. Os absorvedores foram trocados a cada dois dias durante um período de 30 dias, totalizando 15 coletas. Os mesmos foram armazenados em saco plástico na geladeira até o momento da análise.

Para a determinação do $\mathrm{N}$, as espumas foram lavadas em $300 \mathrm{~mL}$ de ácido sulfúrico $(0,0005 \mathrm{M})$. Retirou-se uma alíquota de $50 \mathrm{~mL}$, a qual foi levada para tubo de ensaio. A cada amostra, adicionou-se $5 \mathrm{~mL}$ de $\mathrm{NaOH}$ (50\%) e realizou-se sua destilação pelo uso de um destilador de arraste de vapor. A solução receptora foi o ácido bórico a 5\%, sendo que se utilizou ácido sulfúrico $\left(\mathrm{H}_{2} \mathrm{SO}_{4}\right)$ a $0,025 \mathrm{M}$ para a titulação.

Os dados de perda de $\mathrm{N}$ por volatilização de amônia não foram tratados estatisticamente.

\section{Resultados e Discussão}

Na sequência, as Figuras 1, 2 e 3 apresentam os valores de perda de amônia para cada uma das doses de $\mathrm{N}$ aplicadas, 100, 200 e $300 \mathrm{mg} \mathrm{dm}^{-3}$ respectivamente. A dose de $100 \mathrm{mg} \mathrm{dm}^{-3}$ promoveu o maior pico de volatilização, o qual ocorreu na sexta coleta, realizada no dia 22 de março, sendo dois dias após a segunda aplicação dos tratamentos, motivo pelo qual possa ter ocorrido aumento generalizado de perdas (Figura 1). Primavesi et al. (2001) também encontraram o pico de volatilização em 1 ou 2 dias após a aplicação de fertilizantes. Ainda na mesma coleta, observa-se que as fontes nitrato de amônia e Ajifer foram as que resultaram nas maiores perdas numéricas de amônia por volatilização, em mg vaso ${ }^{-1}$. Nesta mesma coleta, o ponto máximo de perda $\left(18,91 \mathrm{mg}\right.$ vaso $\left.^{-1}\right)$ foi obtido pela fonte Ajifer (fertilizante organomineral: coproduto do glutamato monosódico).

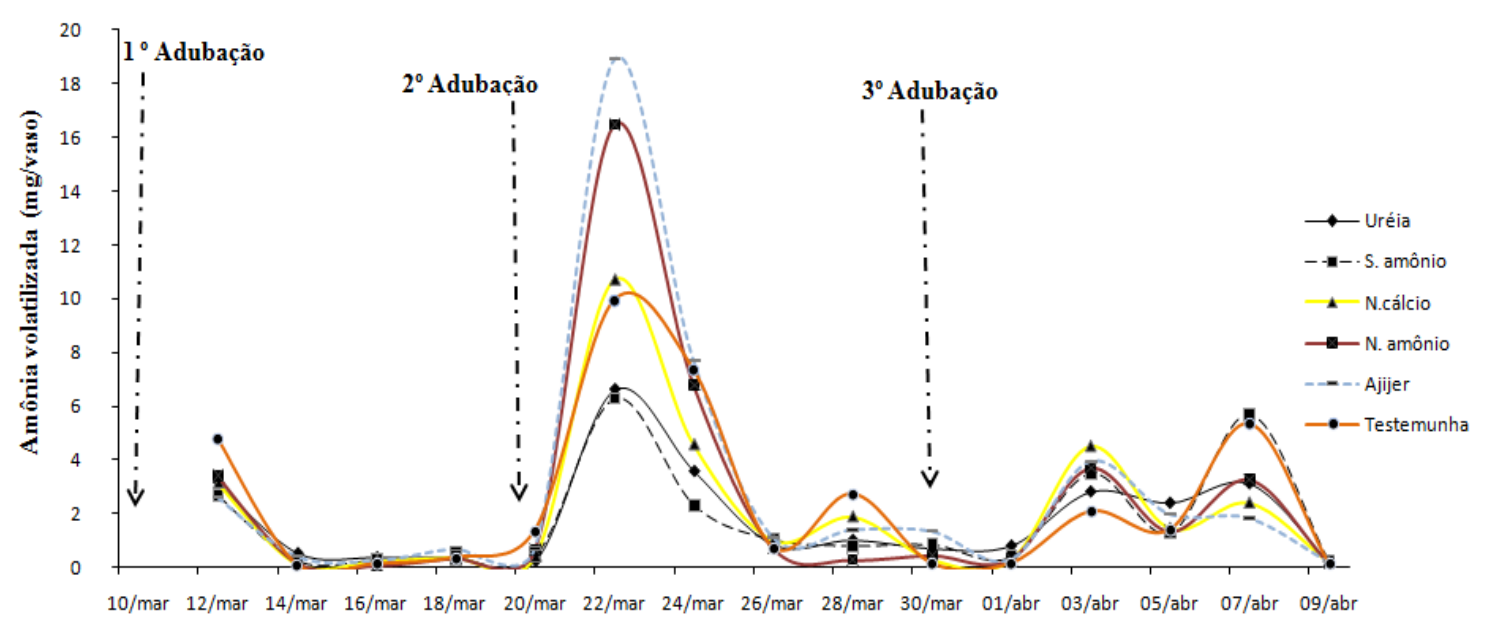

Figura 1. Perdas de amônia por volatilização $\left(\mathrm{mg} \mathrm{vaso}^{-1}\right)$ em função da aplicação de $100 \mathrm{mg} \mathrm{dm}{ }^{-3} \mathrm{de} \mathrm{N}$ em cobertura. Botucatu/SP, 2010. 


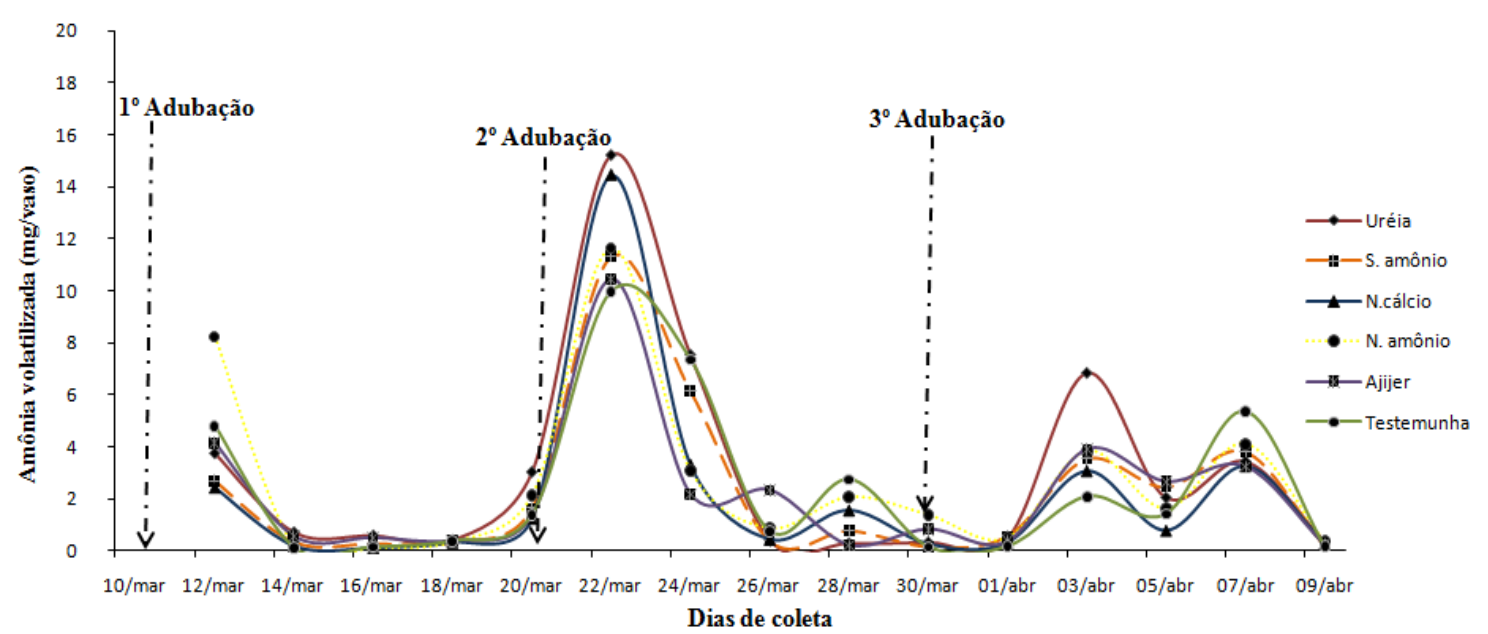

Figura 2. Perdas de amônia por volatilização $\left(\mathrm{mg} \mathrm{vaso}^{-1}\right)$ em função da aplicação de $200 \mathrm{mg} \mathrm{dm} \mathrm{m}^{-3} \mathrm{de} \mathrm{N}$ em cobertura. Botucatu/SP, 2011

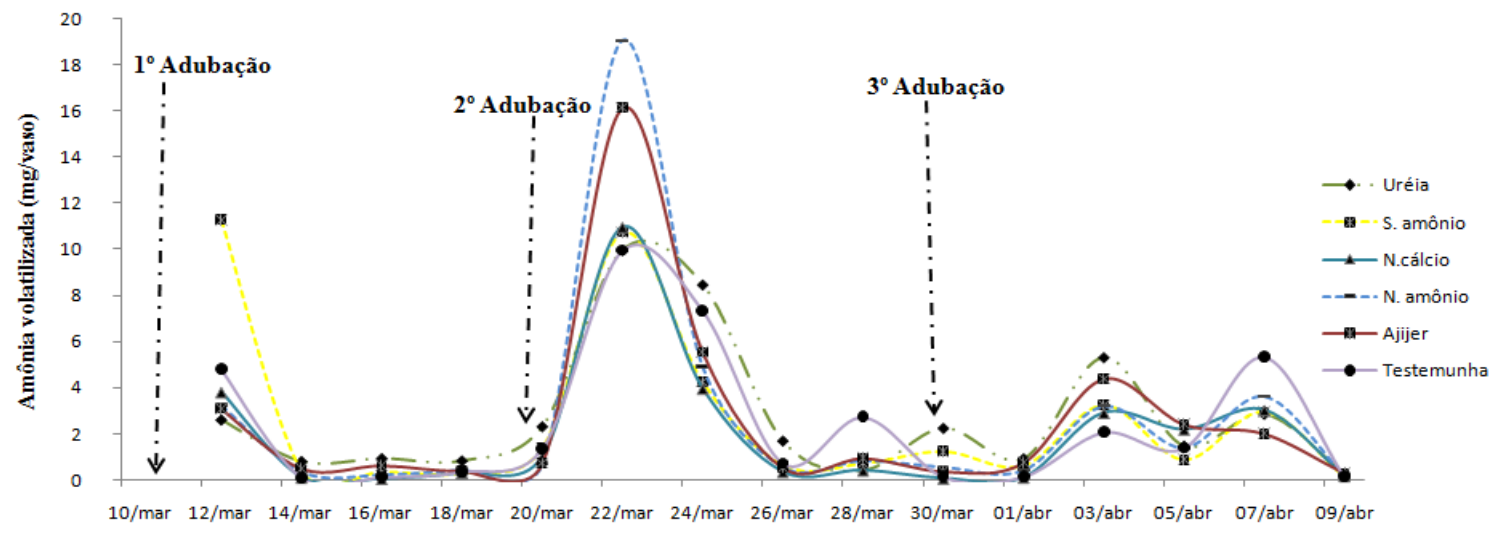

Figura 3. Perdas de amônia por volatilização $\left(\mathrm{mg} \mathrm{vaso}^{-1}\right)$ em função da aplicação de $300 \mathrm{mg} \mathrm{dm}^{-3}$ de $\mathrm{N}$ aplicado em cobertura. Botucatu/SP, 2010.

Na Figura 3, utilizando-se a dose de $200 \mathrm{mg} \mathrm{dm}^{-3}$, a ureia perdeu cerca de $15,21 \mathrm{mg}$ vaso $^{-1}$ de $\mathrm{N}$ na forma de amônia, tendo seu máximo de perda também na sexta coleta, realizada no dia 22 de março, novamente dois dias após a segunda aplicação dos tratamentos.

$\mathrm{Na}$ Figura 3, utilizando a dose de $300 \mathrm{mg} \mathrm{dm}^{-3}$, a fonte que mais perdeu amônia foi o nitrato de amônio $\left(19,03 \mathrm{mg} \mathrm{vaso}^{-1}\right)$, tendo seu máximo de perda também na sexta coleta realizada no dia 22 de março, dois dias após a segunda aplicação dos tratamentos.

O umedecimento do solo, imediatamente após a aplicação da ureia, é mais importante do que a condição de umidade do solo no momento da aplicação (LARA CABEZAS et al., 1997), principalmente quando a ureia é aplicada na superfície e sem incorporação ao solo. A água diminui a volatilização da amônia se for suficiente para diluir a concentração de hidroxilas $\left(\mathrm{OH}^{-}\right)$ao redor dos grânulos de ureia produzidos pela reação de hidrólise, além de proporcionar a incorporação da ureia no solo (LARA CABEZAS et al., 1997). Isso pode explicar porque na dose de $300 \mathrm{mg} \mathrm{dm}^{-3}$ (Figura 3) a ureia foi a fonte que menos perdeu amônia, sendo esta considerada uma fonte que promove perdas significativas por volatilização. A hidrólise da ureia aumenta com a elevação da temperatura até $40^{\circ} \mathrm{C}$ (BREMNER \& MULVANEY, 1978), mas, a hidrólise e as perdas por volatilização de $\mathrm{NH}_{3}$ decrescem rapidamente com o abaixamento da temperatura. De acordo com os dados de temperatura da casa de vegetação, a partir do dia 20/03 (segunda aplicação), a temperatura média variou de $29,5^{\circ} \mathrm{C}$ a $32,5^{\circ} \mathrm{C}$, o que pode ter favorecido a perda por volatilização.

Portanto, as perdas de $\mathrm{N}$ por volatilização de $\mathrm{NH}_{3}$ são afetadas por fatores climáticos e ambientais e favorecidas nas condições do verão brasileiro, nas quais predominam altas temperaturas e umidade.

Acredita-se que, através do processo de mineralização da matéria orgânica, disponibiliza-se até $2 \%$ de $\mathrm{N}$ ao ano para a cultura. Por exemplo, um solo com $3 \%$ de matéria orgânica pode ter $0,15 \%$ de $\mathrm{N}$. Desta forma, é possível que as perdas por volatilização tenham sido influenciadas pelo processo de 
mineralização. Esse fato explicaria, por exemplo, porque a parcela testemunha (sem aplicação de $\mathrm{N}$ em cobertura) apresentou valores consideráveis de perdas de N na forma de amônia (RAIJ, 1991).

\section{Conclusões}

As maiores perdas de nitrogênio por volatilização de amônia ocorreram na segunda época de aplicação para as fontes Ajifer, ureia e nitrato de amônio.

\section{Agradecimentos}

A Fundunesp pelo auxilio concedido ao projeto $\left(\mathrm{N}^{\circ}\right.$ 00028/10).

\section{Referências Bibliográficas}

BOLOGNA, I. R.; FARONI, C. E.; LANGE, A.; TRIVELIN, P. C. O. Perda de nitrogênio pela parte aérea de plantas de trigo. Ciência Rural, Santa Maria-RS, v. 36, n. 4, p. 11061111, 2006.

BITTENCOURT, M. V. L.. Impactos da Agricultura no meioambiente; Principais tendências e desafios (Parte 1). Economia \& Tecnologia. Ano 05. Vol. 18. Julho-Setembro. 2009.

CARVALHO, W. A.; ESPÍNDOLA, C. R.; PACCOLA, A. A. Levantamento de solos da Fazenda Lageado: Estação Experimental "Presidente Médici". Boletim Científico, Botucatu-SP, n. 1, 1983. 95 p.

FERREIRA, D. F. Sisvar. versão 4.2. Lavras-MG: UFLA, 2003. 79 p.
LARA CABEZAS, W. A. R.; KONDORFER, G. H.; MOTTA, S. A. Volatilização de $\mathrm{N}-\mathrm{NH}_{3}$ na cultura de milho: I. Efeito da irrigação e substituição parcial da ureia por sulfato de amônio. Revista Brasileira de Ciência do Solo, ViçosaMG, v. 21, n. 3, p. 481-487, 1997.

MARTINS, D. Classificação climática - Botucatu-SP. Botucatu-SP: UNESP, Faculdade de Ciências Agronômicas, Departamento de Ciências Ambientais, 2003.

NÖNMIK, H. The effect of pellet size on the ammonia loss from urea applied to forest soils. Plant Soil, Dordrecht-UK, v. 39, n. 2n p. 309-318, 1973.

OLIVEIRA, P. P. A.; TRIVELIN, P. C. O.; ALVES, A. C.; LUZ, P. H. C.; HERLING, V. R. Métodos para avaliar as perdas de nitrogênio por volatilização da superfície do solo e por emissão de amônia pela folhagem de Brachiaria brizantha cv. Marandu. São Carlos-SP: Embrapa Pecuária Sudeste, 2008. 41 p. (Boletim de Pesquisa e Desenvolvimento, 16).

PRIMAVESI, O.; CORRÊA, L. A.; PRIMAVESI, A. C.; CANTARELlA, H.; ARMELIN, M. J. A.; SILVA, A. G.; FREITAS, A. R. Adubação com ureia em pastagem de Cynodon dactylon cv Coast-cross sob manejo rotacionado: eficiências e perdas. São Carlos-SP: Embrapa Pecuária Sudeste, 2001. (Circular técnica, 30).

RAIJ, B. van; ANDRADE, J. C.; CANTARELA, H.; QUAGGIO, J. A. Análise química para avaliação da fertilidade de solos tropicais. 1. ed. Campinas-SP: IAC, 2001. $285 \mathrm{p}$.

RAIJ, B. van; CANTARELA, H.; QUAGGIO, J. A.; FURLANI, A. M. C. Recomendação de adubação e calagem para o Estado de São Paulo. 2. ed. Campinas-SP: IAC, 1997. 285 p. (Boletim técnico do Instituto Agronômico de Campinas, n. 100).

RAIJ, B. V. Fertilidade do solo e adubação. Agronômica Ceres Ltda, 1991. p. 71-86. 